\section{An Interplay Between Left Ventricular Wall Thickness and T-Wave Alternans in Pa- tients With Hypertrophic Cardiomyopathy in Predicting Ventricular Tachyarrythmic Events: Is T-Wave Amplitude Difference the Reason? - Reply -}

T-wave alternans (TWA) outcome analysis used in our study ${ }^{1}$ was based on a fully automated spectral analysis method, incorporating previously validated concepts. ${ }^{2,3}$ Inherent to the notion that the magnitude of TWA may be T-wave amplitude dependent is the integral definition of TWA; that is, a beat-to-beat alternation of ECG amplitude and/or morphology within a microvolt range that can be viewed as a periodic oscillation on an every-other-beat basis, reflecting alternations of repolarization at the level of the single cell and subsequent spatial dispersion of repolarization. Given its oscillatory nature, it is the frequency domain analysis and not the amplitude that provides an ideal tool for measuring TWA. Upon aligning the several beats and Fourier transformation, the spectra generated for each point within the $\mathrm{T}$ wave are averaged. ${ }^{4}$ The final result is an aggregate of spectra corresponding to averages of periodic $T$ wave fluctuations across the entire $\mathrm{T}$ wave. The magnitude of TWA can be quantified as the voltage of alternation relative to spectral noise or also thought of as the average difference in T-wave amplitude between consecutive beats. ${ }^{5}$

There is increasing evidence that abnormality in intracellular calcium cycling is responsible for TWA formation, and previous studies of LV hypertrophy (LVH) have suggested that cellular hypertrophy or altered cell-to-cell connections and tissue architecture alter the activation or repolarization sequences and may lead to alternans of action potential duration, particularly at increased heart rates. ${ }^{6,7}$ Relevant to the patients with hypertrophic cardiomyopathy, the TWA is thought to reflect the unique myofibrillar arrangement and/or myocardial fibrosis as the surrogate for electrical instability of the myocardium, rather than reflecting the size of the LV wall thickness (LVWT). ${ }^{8,9}$ In line with this our findings bear significance as to the regional predilection and not the absolute magnitude of the $\mathrm{LVH}$ in itself (LV mass or maximal LVWT) that carries the predictive link of ventricular arrhythmic events and also TWA outcome. ${ }^{1,10}$ Given that TWA is caused by the underlying regional inhomogeneities of ventricular repolarization, it is conceivable that it is the regional distribution of increased LVWT that may have a more relevant role in susceptibility to ventricular tachyarrhythmia over theprominent hypertrophic phenotype. Increased inferior LVWT may link with amassed regional fibrosis, ${ }^{11}$ or myofibrillar disarray, and its direct involvement in arrhythmia vulnerability and/or TWA outcomes needs to be ascertained in future studies.

\section{References}

1. Puntmann VO, Yap YG, McKenna W, Camm J. T-wave alternans and left ventricular wall thickness in predicting arrhythmic risk in patients with hypertrophic cardiomyopathy. Circ J 2010; 74: 1197-1204.

2. Bloomfield DM, Hohnloser SH, Cohen RJ. Interpretation and classification of microvolt T-wave alternans tests. J Cardiovasc Electrophysiol 2002; 13: 502-512.

3. Richter S, Duray G, Hohnloser SH. How to analyze T-wave alternans. Heart Rhythm 2005; 2: 1268-1271.

4. Narayan SM, Smith JM. Exploiting rate hysteresis in repolarization alternans to optimize the sensitivity and specificity for ventricular tachycardia. J Am Coll Cardiol 2000; 35: 1485-1492.

5. Narayan SM. T-wave alternans and the susceptibility to ventricular arrhythmias [State of the Art paper]. J Am Coll Cardiol 2006; 47: $269-281$.

6. Narayan SM, Smith JM, Lindsay BD, Cain ME, Dávila-Román VG. Relation of T-wave alternans to regional left ventricular dysfunction and eccentric hypertrophy secondary to coronary heart disease. Am J Cardiol 2006; 97: 775-780.

7. Chinushi M, Restivo M, Caref EB, El-Sherif N. Electrophysiological basis of arrhythmogenicity of QT/T alternans in the long-QT syndrome: Tridimensional analysis of the kinetics of cardiac repolarization. Circ Res 1998; 83: 614-628.

8. Kon-No Y, Watanabe J, Koseki Y, Koyama J, Yamada A, Toda S, et al. Microvolt T-wave alternans in human hypertrophy: Electrical instability and abnormal myocardial arrangement. $J$ Cardiovasc Electrophysiol 2001; 12: 759-763.

9. Kuroda N, Ohnishi Y, Yoshida A, Kimura A, Yokoyama M. Clinical significance of T-wave alternans in hypertrophic cardiomyopathy. Circ J 2002; 66: 457-462.

10. Puntmann VO, Yap YG, McKenna W, Camm AJ. Significance of maximal and regional left ventricular wall thickness in association with arrhythmic events in patients with hypertrophic cardiomyopathy. Circ J 2010; 74: 531-537.

11. Puntmann VO, Jahnke C, Gebker R, Schnackenburg B, Fox KF, Fleck E, et al. Usefulness of magnetic resonance imaging to distinguish hypertensive and hypertrophic cardiomyopathy. Am J Cardiol 2010 (in press).

Valentina O. Puntmann, MD, PhD

St George's, University of London, Cranmer Terrace, London, UK

(Released online July 14, 2010) 